\title{
Estudo florístico do componente herbáceo e relação com solos em áreas de caatinga do embasamento cristalino e bacia sedimentar, Petrolândia, PE, Brasil ${ }^{1}$
}

\author{
Kleber Andrade da Silva ${ }^{2,4}$, Elcida de Lima Araújo² e Elba Maria Nogueira Ferraz ${ }^{3}$
}

Recebido em 11/06/2007. Aceito em 26/05/2008

\begin{abstract}
RESUMO - (Estudo florístico do componente herbáceo e relação com solos em áreas de caatinga do embasamento cristalino e bacia sedimentar, Petrolândia, PE, Brasil). Realizou-se um levantamento florístico em áreas de caatinga do embasamento cristalino e de bacia sedimentar, em Petrolândia, Pernambuco, objetivando-se identificar diferenças na composição, hábito e forma de vida das herbáceas entre as duas áreas. Foram coletadas amostras de solo nas profundidades de 0 a $20 \mathrm{~cm}$ e de 20 a $40 \mathrm{~cm}$. Foram estabelecidas 100 parcelas de $1 \times 1 \mathrm{~m}$ em cada área e coletadas todas as espécies herbáceas. De modo geral, o solo da área do embasamento cristalino foi mais fértil, raso, com maior capacidade de reter água e maior porcentagem de argila. Os números de famílias, gêneros e espécies das áreas da bacia sedimentar e do embasamento cristalino foram 32, 62 e 78 e 31, 53 e 69, respectivamente. A similaridade florística entre as áreas foi alta (75\%). A análise de agrupamento mostrou que os arranjos florísticos entre as parcelas amostradas são mais semelhantes dentro de cada área que entre áreas, sugerindo a existência de grupos distintos. Tipo de solo parece não ser um fator decisivo na similaridade florística, hábito e proporção de forma de vida das herbáceas da vegetação da caatinga quando as áreas são próximas, mas pode influenciar a ocorrência e a freqüência de algumas espécies.
\end{abstract}

Palavras-chave: caatinga, florística, formas de vida, herbáceas, solos

\begin{abstract}
Herbaceous floristic study and relationship with soil of crystalline shield and sedimentary basin caatinga areas at Petrolândia, Pernambuco State, Brazil). Floristic surveys were carried out in two close caatinga areas, one on the crystalline shield and the other on a sedimentary basin, both at Petrolândia, Pernambuco, Brazil, with the objective of identifying differences between the two areas in composition, habit and life form of the herbaceous flora. One hundred $1 \times 1 \mathrm{~m}$ plots were established in each area and all the herbaceous species were identified. Soil samples were collected at 0-20 and 20-40 cm depths. In general, the soil in the crystalline shield area was shallower, had greater clay content, with higher water holding capacity, and was more fertile. The number of families, genera and species in the sedimentary basin and crystalline shield areas were 32, 62 and 78 and 31, 53 and 69, respectively. The floristic similarity between the areas was high (75\%). The clustering analysis showed that the floristic arrangements between the sampled plots are more similar within the areas than between areas, suggesting the existence of distinct groups. Soil type seems not to be a factor determining the floristic similarity, habit or life forms of the caatinga vegetation herbs when the areas are near, but it may influence the occurrence and the frequency of some species.
\end{abstract}

Key words: caatinga, floristic, herbaceous, life form, soil

\section{Introdução}

Tipos de solo e clima são fatores abióticos que influenciam a distribuição de plantas lenhosas no ecossistema caatinga (Andrade-Lima 1981; Prado 2003), pois a composição florística e a estrutura do componente lenhoso de áreas de caatinga instaladas sobre o embasamento cristalino (Araújo et al. 1995; Ferraz et al. 1998; Alcoforado Filho et al. 2003) têm apresentado diferenças em relação às ocorrentes sobre bacias sedimentares (Oliveira et al. 1997; Rodal et al. 1999; Lemos \& Rodal 2002).

No entanto, nenhum estudo verificou se o solo poderia influenciar a distribuição de plantas herbáceas na caatinga. Esta lacuna pode ser justificada pelo fato da flora herbácea ter sido pouco considerada nos estudos florísticos e fitossociológicos (Rodal et al. 1999; Araújo et al. 2002a; Araújo et al. 2005; Pessoa et al. 2004; Rodal et al. 2005; Reis et al. 2006; Costa et al. 2007; Feitoza et al. 2008; M.O.M Feitoza, dados não

1 Parte da Dissertação de Mestrado do primeiro Autor, Programa de Pós-Graduação em Botânica da UFRPE

2 Universidade Federal Rural de Pernambuco, Departamento de Biologia, Área de Botânica, Laboratório de Ecologia Vegetal dos Ecossistemas Naturais, Av. Dom Manoel de Medeiros s.n., Dois Irmãos, 52171-900 Recife, PE, Brasil

3 Centro Federal de Educação Tecnológica de Pernambuco, Av. Professor Luiz Freire 500, Cidade Universitária, 50740-540 Recife, PE, Brasil

4 Autor para correspondência: kleberandradedasilva@ hotmail.com 
publicados), apesar de sua elevada diversidade (Araújo et al. 2007) e de seu diversificado valor econômico (Pereira et al. 1989; Lorenzon et al. 2003; Araújo Filho et al. 2002; Araújo et al. 2002a; Albuquerque et al. 2007).

Vale ainda destacar que o componente herbáceo na caatinga apresenta importante papel ecológico, contribuindo para a manutenção da flora lenhosa local. De acordo com Araújo (2003) e M.O.M. Feitoza (dados não publicados) solos da caatinga recobertos por plantas herbáceas são mais protegidos contra processos erosivos e mantêm temperatura e umidade mais elevadas, quando comparados a solos descobertos, funcionando como uma câmara de germinação natural quente e úmida. Em adição, essas autoras registram que o sistema radicular das ervas geralmente se entrelaça na camada superficial do solo, formando uma malha que auxilia na retenção de sementes tanto de plantas lenhosas quanto de herbáceas, o que favorece o processo de regeneração das populações vegetais.

Diante do exposto, este estudo objetivou descrever as características físico-químicas dos solos de uma área sedimentar e de uma área cristalina da caatinga, identificar a composição florística do componente herbáceo, verificar a existência de diferenças na composição florística entre as áreas e verificar a existência de relações solo-vegetação nessas áreas.

\section{Material e métodos}

Caracterização e seleção das áreas do estudo - O estudo foi realizado em duas áreas de caatinga, em Petrolândia, Pernambuco. Este município é, em parte, cortado pelo rio São Francisco, que segundo Brasil (1983) apresenta seções distintas, de acordo com sua morfologia, gradiente de declividade, velocidade de lâmina de água e capacidade de transporte de sedimentos. A primeira das seções abrange desde a Barragem de Sobradinho até a cidade de Petrolândia, onde o rio entalha rochas metamórficas do Pré-Cambriano e sedimentos cretácicos da bacia Tucano-Jatobá.

Como a flora herbácea da caatinga é efêmera e sensível às variações do regime pluviométrico (Araújo et al. 2002a; Reis et al. 2006; Araújo et al. 2007), tomou-se o cuidado de selecionar áreas próximas entre si (cerca de 1,5 km) para amostragem, visando diminuir a influência de variações climáticas locais sobre seu conjunto florístico. As duas áreas estão situadas no Distrito de Mundo Novo, sendo que uma ocorre sobre solos arenosos do domínio geomorfológico das bacias e coberturas sedimentares (coordenadas 9 04 '57'S e $38^{\circ} 13^{\prime} 47^{\prime \prime} \mathrm{W}$, com $432 \mathrm{~m}$ de altitude) e a outra sobre solos pedregosos do domínio do escudo exposto (nas coordenadas $9^{\circ} 05^{\prime} 27^{\prime \prime} \mathrm{S}$ e $38^{\circ} 13^{\prime} 43^{\prime \prime} \mathrm{W}$, com $430 \mathrm{~m}$ de altitude), de acordo com a classificação de Brasil (1983) e serão aqui chamadas de áreas sedimentar e cristalina, respectivamente. São drenadas pelo riacho do Salgado, que nasce na Serra de Tacaratu e deságua no rio São Francisco e apresentam clima do tipo Bshw, semi-árido quente, segundo a classificação de Köppen, com total pluviométrico anual em torno de $435 \mathrm{~mm}$ e chuvas concentradas nos meses de janeiro a maio. A temperatura, a umidade relativa e a evaporação potencial média anual são de $25{ }^{\circ} \mathrm{C}, 55 \%$ e $3.008 \mathrm{~mm}$, respectivamente (FIAM/DI 1986; Perazzo 2002).

As duas áreas pertencem a uma propriedade particular e, segundo os proprietários, nos últimos 20 anos os trechos selecionados e amostrados não foram explorados por atividades de agricultura e pecuária extensiva. A flora lenhosa da área sedimentar caracterizase pela presença de espécies como: Amburana cearensis (Allemão) A.C. Sm., Anadenanthera colubrina (Vell.) Brenan, Aspidosperma pyrifolium Mart., Caesalpinia microphylla Mart. ex G. Don, Bauhinia cheilantha (Bong.) Steud., Cereus jamacaru DC., Cnidoscolus phyllacanthus (Müll. Arg.) Pax \& L. Hoffm., Commiphora leptophloeos (Mart.) J.B. Gillett, Croton rhamnifolioides Pax \& K. Hoffm., Guettarda angelica Mart. ex Müll. Arg., Jatropha mollissima (Pohl) Baill., Jatropha ribifolia (Pohl) Baill., Lantana camara L., Maytenus rigida Mart., Opuntia palmadora Britton \& Rose, Spondias tuberosa Arruda, Rhamnidium elaeocarpum Reissek, Rolliniopsis leptopetala (R.E. Fr.) Saff., Ziziphus joazeiro Mart. e espécies de Myrtaceae e Rutaceae. De uma maneira geral, a fisionomia da área sedimentar é arbustivo-arbórea aberta e de porte baixo, com extensas áreas de penetração de luz. Nos trechos mais abertos, merecem destaque Jatropha mollissima e $J$. ribifolia, que formam populações numerosas.

Já a flora lenhosa da área cristalina caracteriza-se pela presença de Caesalpinia pyramidalis Tul., Capparis flexuosa (L.) L., Commiphora leptophloeos (Mart.) J.B. Gillett, Croton argyrophylloides Müll. Arg., Guettarda angelica Mart. ex Müll. Arg., Myracrodruon urundeuva Allemão, Opuntia palmadora Britton \& Rose, Pilosocereus gounellei (F.A.C. Weber) Byles \& G.D. Rowley, Rhamnidium elaeocarpum Reissek, Syagrus coronata (Mart.) Becc., Sideroxylon obtusifolium (Humb. ex Roem. \& Schult.) T.D. Penn. e Ziziphus joazeiro Mart. Dentre estas espécies, $C$. argyrophylloides forma população numerosa. A fisionomia da área cristalina é arbustivo-arbórea de porte mais elevado quando comparada à da área sedimentar. Diferentemente da área sedimentar, na área cristalina existem muitos afloramentos rochosos, onde espécies das famílias Bromeliaceae (principalmente Encholirium spectabile Mart. ex Schult. f.) e Cactaceae se estabelecem e 
marcam a fisionomia da área. As copas das espécies arbóreas formam um dossel quase contínuo, tornando a área mais fechada e com menor penetração de luz em relação à área sedimentar.

Coleta do solo - O solo da área sedimentar apresenta trechos descobertos e cobertos com serrapilheira, enquanto na área cristalina, onde não ocorrem afloramentos rochosos, o solo é quase totalmente recoberto por serrapilheira. Em ambas as áreas foram retiradas cinco amostras simples de solo. As amostras foram feitas a uma distância de aproximadamente $25 \mathrm{~m}$ entre si, nas profundidades de 0 a $20 \mathrm{~cm}$ e de 20 a $40 \mathrm{~cm}$, todavia na área cristalina, três das amostras chegaram apenas a $30 \mathrm{~cm}$ de profundidade, devido à presença de rochas. Posteriormente, as amostras de solo das áreas sedimentar e cristalina, de cada profundidade e de cada trecho, foram misturadas para as análises física e química, resultando em seis amostras compostas. As análises foram realizadas nos laboratórios de Física e de Química do Solo do Departamento de Agronomia da Universidade Federal Rural de Pernambuco (UFRPE).

Amostragem do componente herbáceo - Foram traçados dez transectos perpendiculares e eqüidistantes em $10 \mathrm{~m}$, com início a $5 \mathrm{~m}$ da borda em ambas as áreas. Ao longo de cada transecto foram alocadas dez parcelas de $1 \times 1 \mathrm{~m}$ a uma distância fixa de $5 \mathrm{~m}$, totalizando 100 parcelas em cada área.

Inicialmente, todas as ervas não floridas das parcelas foram marcadas e coletadas amostras das que se apresentavam floridas nas parcelas e/ou de indivíduos próximos. Ervas amostradas apenas fora das parcelas foram indicadas como observadas na lista florística. A partir de então, foram feitas visitas mensais às parcelas amostradas e realizadas caminhadas por toda a área para coleta de material reprodutivo das espécies que não estavam floridas no início da amostragem, com o intuito de ampliar o esforço de coleta.

Como as herbáceas apresentavam diferenças quanto à visibilidade ao nível do solo e ao teor de lignificação do caule, as mesmas foram classificadas em: 1) erva - planta de caule não lignificado e de cor verde ou planta que ao nível do solo tinha o sistema aéreo representado diretamente pelas folhas. Neste último caso, os caules das ervas geralmente não eram visíveis ao nível do solo, mas quando visíveis eram pequenos, decubentes e rizomatosos, fato registrado apenas para espécies de Bromeliaceae e Liliaceae; 2) erva/subarbustiva - planta que apresenta caule com baixo nível de lignificação e de cor variando de verde a amarronzado; 3 ) erva/trepadeira - planta que apresenta caule verde, não lignificado e hábito trepador, podendo atingir grandes alturas.

Em campo, algumas plântulas de herbáceas foram confundidas com plântulas de espécies lenhosas devido à semelhança morfológica existente. Para corrigir o problema, indivíduos dessas espécies foram coletados, plantados em sacos de polietileno contendo solo do local, transportados para casa de vegetação e monitorados até obtenção de material reprodutivo. Após correta identificação, as plântulas das lenhosas foram eliminadas da análise.

As principais formas de vida existentes em cada área foram identificadas com base no sistema de classificação de Raunkiaer (Raunkiaer 1934), visando verificar se os tipos de solos influenciariam na freqüência de formas de vida do componente herbáceo. Para isto, na chegada da estação desfavorável à sobrevivência das plantas (sete meses de seca) foi observado se indivíduos das espécies permaneciam vivos na área, se lançavam sementes no solo e morriam, ou se perdiam o sistema aéreo mas mantinham gemas, bulbos ou rizomas no solo, ou abaixo deste, que permitisse a regeneração da população na estação chuvosa subsequiente. Para tanto, foram feitas escavações na base da planta.

Análises florísticas - O material botânico foi herborizado seguindo as técnicas usuais de preparação, secagem e montagem de exsicatas (Mori et al. 1989). A identificação taxonômica foi realizada por comparações com exsicatas depositadas nos herbários Prof. Vasconcelos Sobrinho (PEUFR) e Dárdano de Andrade Lima (IPA) e com o auxilio de chaves taxonômicas e literatura específica. Exsicatas das espécies com identificação problemática ou duvidosa foram enviadas para especialistas. A grafia do nome das espécies foi verificada a partir de consulta ao Index Kewensis (www.ipni.org/ipni/ plantnamesearchpage.do) e ao banco de dados do Missouri Botanical Garden's VAST - MOBOT (www.mobot.mobot.org/W3T/Search/vast.html). A abreviação dos nomes de autores das espécies foi feita por consulta ao Brummit \& Powell (1992) e ao MOBOT. Após identificação, as exsicatas coletadas por K.A.Silva foram incorporadas ao acervo do Herbário Professor Vasconcelos Sobrinho - PEUFR e duplicatas enviadas para o acervo do Herbário Dárdano de Andrade Lima - IPA.

Para caracterizar a flora herbácea foi elaborada uma lista geral de espécies para as duas áreas, seguindo o sistema de classificação de Cronquist (1988). Para cada espécie são fornecidas informações sobre o número do registro (Herbário PEUFR), freqüência de ocorrência por área amostrada (sedimentar e cristalino), hábito e forma de vida. Diferenças na freqüência das espécies nas parcelas e na freqüência de formas de vida entre as áreas sedimentar e cristalina foram avaliadas pelo teste Quiquadrado (Zar 1996).

Foi calculada a similaridade florística entre as áreas sedimentar e cristalina, utilizando-se o índice de Sørensen (Krebs 1989). Além disso, foi elaborada uma matriz de 
presença/ausência das espécies amostradas nas parcelas nas áreas sedimentar (parcelas de 1 a 100) e cristalina (parcelas de 101 a 200). A partir desta matriz, foi testada a similaridade florística entre as áreas através da análise de agrupamento, utilizando o índice de distância BrayCurtis e a técnica de ligação de média de grupo (UPGMA). A análise foi feita pelo programa PC ORD4 System (McCune \& Mefford 1999). A casualidade das ligações da análise de agrupamento foi avaliada pelo teste Monte Carlo, a 5\% de probabilidade pelo programa RandMat 1.0 (http://eco.ib.usp.br/labmar).

\section{Resultados e discussão}

Caracterização dos solos - Os solos dos trechos descobertos e cobertos com serrapilheira na chamada área sedimentar enquadram-se na classe textural arenosa. De uma maneira geral, as proporções de areia, argila e silte foram semelhantes entre os dois locais e entre as profundidades de 0 a $20 \mathrm{~cm}$ e $20 \mathrm{a} 40 \mathrm{~cm}$. Já na área designada cristalina, o solo foi enquadrado na classe textural franco-arenosa, com menores proporções de areia e maiores de argila, nas duas profundidades, quando comparadas às proporções da área sedimentar (Tab. 1). Além de mais argiloso e pedregoso, o solo da área cristalina apresentou maior capacidade de retenção de água em relação ao solo da área sedimentar, apesar deste último ser mais profundo. Quanto à fertilidade, o solo da área cristalina apresentou-se mais fértil, sobretudo em relação ao fósforo (Tab. 1). Tais características são relatadas como freqüentes em solos das áreas sedimentar e cristalina (Jacomine et al. 1973; Souza et al. 1994; Sampaio \& Gamarra-Rojas 2003).

Especificamente na área sedimentar, a camada mais superficial do solo nos trechos descobertos e cobertos por serrapilheira apresentou teores mais elevados de $\mathrm{K}$, $\mathrm{Ca}, \mathrm{H}, \mathrm{P}$ e C quando comparada à profundidade de $20 \mathrm{a}$ $40 \mathrm{~cm}$, mas os trechos cobertos por serrapilheira tendiam ser mais férteis (Tab. 1). Tal fato foi registrado como importante para o crescimento de ervas em outras florestas secas do mundo, onde, segundo Fuller (1999), apesar de disponibilidade de água ser um fator de fundamental importância para manutenção da camada herbácea, em áreas de savana da Zâmbia, o sombreamento proporcionado pelas copas das árvores e a serrapilheira proveniente da queda das folhas das árvores na estação seca, possibilitava uma interação positiva que favorecia o crescimento das ervas. Apesar de não mensurado, isto também foi observado na área deste estudo, pois onde houve presença de serrapilheira na área sedimentar a densidade das populações e a altura média das herbáceas foram maiores (cerca de $25 \mathrm{~cm}$ ), conferindo maior grau de recobrimento ao solo. Nos trechos sem serrapilheira, a fisionomia do componente herbáceo foi marcada pela ocorrência de Evolvulus frankenioides Moric., uma herbácea prostada que recobria extensas áreas do solo.

Apesar da proximidade entre as áreas, foi observado que a área cristalina apresentava menor luminosidade com pequenas clareiras formadas pela ausência de copas de plantas lenhosas em alguns trechos. As parcelas estabelecidas em trechos não sombreados apresentavam populações herbáceas mais numerosas, conferindo maior recobrimento ao solo. Este comportamento apresentase oposto ao encontrado na área sedimentar estudada, mas assemelha-se aos registrados por Sampaio (2003) e por M.O.M. Feitoza (dados não publicados) para áreas não sombreadas da caatinga, onde a biomassa herbácea é maior. Isto aponta existir modelos de interações entre plantas lenhosas e herbáceas na caatinga que precisam

Tabela 1. Variáveis físicas e químicas analisadas das amostras de solo coletadas nas profundidades de 0 a $20 \mathrm{~cm}$ e 20 a $40 \mathrm{~cm}$ das áreas sedimentar e cristalina, em Petrolândia, PE, Brasil.

\begin{tabular}{|c|c|c|c|c|c|c|}
\hline \multirow[t]{3}{*}{ Variáveis } & \multicolumn{4}{|c|}{ Sedimentar } & \multicolumn{2}{|c|}{ Cristalino } \\
\hline & \multicolumn{2}{|c|}{ Sem serrapilheira } & \multicolumn{2}{|c|}{ Coberto com serrapilheira } & \multirow[t]{2}{*}{$0-20$} & \multirow[t]{2}{*}{$20-40$} \\
\hline & $0-20$ & $20-40$ & $0-20$ & $20-40$ & & \\
\hline Areia $(\%)$ & 91,10 & 90,60 & 88,60 & 88,80 & 83,10 & 83,60 \\
\hline Argila (\%) & 4,40 & 5,40 & 4,90 & 5,40 & 9,40 & 10,40 \\
\hline Silte $(\%)$ & 4,50 & 4,00 & 6,50 & 6,00 & 7,50 & 6,00 \\
\hline Capacidade de campo (\%) & 7,77 & 6,74 & 8,96 & 7,92 & 12,16 & 10,34 \\
\hline $\mathrm{pH}$ (água - 1:2,5) & 5,40 & 5,80 & 5,80 & 6,30 & 6,00 & 6,30 \\
\hline $\mathrm{K}^{+}\left(\mathrm{cmol}_{\mathrm{c}} \cdot \mathrm{dm}^{-3}\right)$ & 0,03 & 0,04 & 0,08 & 0,06 & 0,23 & 0,17 \\
\hline $\mathrm{Ca}^{2+}\left(\mathrm{cmol}_{\mathrm{c}} \cdot \mathrm{dm}^{-3}\right)$ & 1,00 & 0,75 & 3,65 & 1,40 & 3,70 & 2,50 \\
\hline $\mathrm{Mg}^{2+}\left(\mathrm{cmol}_{\mathrm{c}} \cdot \mathrm{dm}^{-3}\right)$ & 1,35 & 1,05 & 0,95 & 1,20 & 2,30 & 1,40 \\
\hline $\mathrm{H}^{+}\left(\mathrm{cmol}_{\mathrm{c}} \cdot \mathrm{dm}^{-3}\right)$ & 0,18 & 0,18 & 1,06 & 0,28 & 1,35 & 1,27 \\
\hline $\mathrm{Al}^{3+}\left(\mathrm{cmol}_{\mathrm{c}} \cdot \mathrm{dm}^{-3}\right)$ & 0,15 & 0,15 & 0,10 & 0,05 & 0,05 & 0,05 \\
\hline $\mathrm{P}$ disponível $\left(\mathrm{mg} \cdot \mathrm{dm}^{-3}\right)$ & 2,70 & 2,20 & 6,70 & 1,00 & 96,00 & 93,00 \\
\hline Carbono orgânico $\left(\mathrm{g} \cdot \mathrm{kg}^{-1}\right)$ & 0,82 & 0,61 & 7,44 & 0,87 & 10,07 & 0,87 \\
\hline
\end{tabular}


104 Silva, Araújo \& Ferraz: Estudo florístico do componente herbáceo e relação com solos em áreas de caatinga...

ser melhor investigados, o que já vem sedo evidenciado para outros ambientes secos do mundo (Knoop \& Walker 1985; Fuller 1999; Peters 2002).

Caracterização e similaridade florística - A flora herbácea das áreas sedimentar e cristalina esteve representada por 95 espécies, pertencentes a 39 famílias e 75 gêneros (Tab. 2). Das 95 espécies, cinco foram identificadas apenas até gênero, e uma apenas até família. Nenhuma espécie de Briófita ou de Pteridófita foi observada na área, apesar destes grupos ocorrerem em outras áreas de caatinga (Araújo et al. 2005; Reis et al. 2006). Isoladamente, a área sedimentar apresentou 78 espécies, distribuídas por 62 gêneros e 32 famílias. Já a área cristalina, apresentou 69 espécies, distribuídas por 53 gêneros e 31 famílias (Tab. 2). Comparativamente, não houve diferença significativa na riqueza de espécies amostradas nas parcelas entre as áreas, a qual esteve

Tabela 2. Lista florística das famílias e espécies de ervas encontradas nas áreas cristalina (Cris) e sedimentar (Sed) em uma área de caatinga de Petrolândia, PE, Brasil. $\mathrm{N}^{\circ}$ do registro = número do registro das exsicatas depositadas no herbário Prof. Vasconcelos Sobrinho (PEUFR); $\mathrm{Hab}=$ hábito; $\mathrm{FV}=$ forma de vida; $\mathrm{Er}=$ erva; $\mathrm{Er} / \mathrm{T}=$ erva/trepadeira; $\mathrm{Er} / \mathrm{S}=$ erva/subarbusto; $\mathrm{T}=$ terófita $; \mathrm{C}=\mathrm{caméfita} ; \mathrm{CS}=\mathrm{Caméfita}$ suculenta; $\mathrm{G}=$ geófita; * = espécies apenas observadas. Os números das colunas das áreas cristalina e sedimentar indicam o número de parcelas com registro da espécie. Letras diferentes numa mesma linha entre as colunas indicam diferença significativa na freqüência pelo teste do Qui-quadrado a 5\% de probabilidade.

\begin{tabular}{|c|c|c|c|c|c|}
\hline Família/Espécie & $\mathrm{N}^{\circ}$ do Registro & Cris & Sed & $\mathrm{Hab}$ & FV \\
\hline \multicolumn{6}{|l|}{ AMARANTHACEAE } \\
\hline Alternanthera tenella Colla & $45397 ; 45396$ & $60 \mathrm{a}$ & $56 a$ & Er & $\mathrm{T}$ \\
\hline Amaranthus viridis L. & $45398 ; 45399$ & 3 & 4 & Er & $\mathrm{T}$ \\
\hline \multicolumn{6}{|l|}{ ARISTOLOCHIACEAE } \\
\hline Aristolochia birostris Duch. & $45401 ; 45402$ & $11 \mathrm{a}$ & $9 \mathrm{a}$ & $\mathrm{Er} / \mathrm{T}$ & $\mathrm{C}$ \\
\hline \multicolumn{6}{|l|}{ ASCLEPIADACEAE } \\
\hline Matelea maritima subsp. ganglinosa (Vell.) Fontella & 45403 & * & & $\mathrm{Er} / \mathrm{T}$ & $\mathrm{C}$ \\
\hline \multicolumn{6}{|l|}{ ASTERACEAE } \\
\hline Acanthospermum hispidum DC. & 45820 & & $*$ & $\mathrm{Er} / \mathrm{S}$ & $\mathrm{T}$ \\
\hline Blainvillea rhomboidea Cass. & $45404 ; 45405$ & $8 \mathrm{a}$ & $7 a$ & $\mathrm{Er} / \mathrm{S}$ & $\mathrm{T}$ \\
\hline Centratherum punctatum Cass. & 45824 & & $*$ & $\mathrm{Er} / \mathrm{S}$ & $\mathrm{T}$ \\
\hline Emilia sonchifolia (L.) DC. & $45643 ; 45825$ & $*$ & $*$ & $\mathrm{Er}$ & $\mathrm{T}$ \\
\hline Tridax procumbens $\mathrm{L}$. & $45406 ; 45658$ & 6 & * & Er & $\mathrm{T}$ \\
\hline \multicolumn{6}{|l|}{ BIGNONIACEAE } \\
\hline Anemopaegma laeve DC. & 45407 & $*$ & & $\mathrm{Er} / \mathrm{T}$ & $\mathrm{C}$ \\
\hline \multicolumn{6}{|l|}{ BORAGINACEAE } \\
\hline Heliotropium angiospermum Murray & $4548 ; 45409$ & $13 \mathrm{a}$ & $2 b$ & Er & $\mathrm{T}$ \\
\hline H. procumbens Mill. & 45410 & 6 & & $\mathrm{Er}$ & $\mathrm{T}$ \\
\hline \multicolumn{6}{|l|}{ BROMELIACEAE } \\
\hline Encholirium spectabile Mart. ex Schult. f. & 45411 & 11 & & $\mathrm{Er}$ & $\mathrm{C}$ \\
\hline Hohenbergia catingae Ule & 45412 & 7 & & $\mathrm{Er}$ & $\mathrm{C}$ \\
\hline Neoglaziovia variegata (Arruda) Mez & 45413 & 6 & & Er & $\mathrm{C}$ \\
\hline \multicolumn{6}{|l|}{ CACTACEAE } \\
\hline Opuntia inamoena $\mathrm{K}$. Schum. & $45414 ; 45818$ & $11 \mathrm{a}$ & $14 \mathrm{a}$ & Er & $\mathrm{CS}$ \\
\hline Melocactus bahiensis (Britton \& Rose) Luetzelb. & 45482 & 3 & & $\mathrm{Er}$ & CS \\
\hline \multicolumn{6}{|l|}{ CAESALPINIACEAE } \\
\hline Chamaecrista trichopoda (Benth.) Britton \& Rose ex Britton \& Killip. & 45655 & & 6 & Er & $\mathrm{T}$ \\
\hline \multicolumn{6}{|l|}{ CAPPARACEAE } \\
\hline Cleome diffusa Banks ex DC. & $45416 ; 45415$ & $70 \mathrm{a}$ & $18 b$ & Er & $\mathrm{T}$ \\
\hline C. guianensis Aubl. & 45417 & & 7 & Er & $\mathrm{T}$ \\
\hline C. rotundifolia (Mart. \& Zucc.) H.H. Iltis & $45419 ; 45418$ & $3 b$ & $55 \mathrm{a}$ & Er & $\mathrm{T}$ \\
\hline \multicolumn{6}{|l|}{ CAPPARACEAE } \\
\hline Dactylaena micrantha Schrad. ex Schult. \& Schult. f. & 45420 & & 6 & Er & $\mathrm{T}$ \\
\hline \multicolumn{6}{|l|}{ COMMELINACEAE } \\
\hline Callisia repens (Jacq.) L. & $45421 ; 45422$ & $55 \mathrm{a}$ & $5 b$ & Er & $\mathrm{T}$ \\
\hline Commelina obliqua Vahl & $45423 ; 45653$ & $20 b$ & $38 \mathrm{a}$ & Er & $\mathrm{T}$ \\
\hline \multicolumn{6}{|l|}{ CONVOLVULACEAE } \\
\hline Evolvulus frankenioides Moric. & $45425 ; 45424$ & $3 b$ & $72 \mathrm{a}$ & $\mathrm{Er} / \mathrm{S}$ & $\mathrm{C}$ \\
\hline Ipomoea aristolochiifolia G. Don & 45429 & $*$ & & $\mathrm{Er} / \mathrm{T}$ & $\mathrm{G}$ \\
\hline I. brasiliana (C. Martius) Meisn. & $45428 ; 45427$ & $34 \mathrm{a}$ & $52 \mathrm{a}$ & $\mathrm{Er} / \mathrm{T}$ & $\mathrm{G}$ \\
\hline I. rosea Choisy & $45433 ; 45426$ & 4 & 3 & $\mathrm{Er} / \mathrm{T}$ & $\mathrm{C}$ \\
\hline Merremia aegyptia (L.) Urb. & $45821 ; 45432$ & 2 & 1 & $\mathrm{Er} / \mathrm{T}$ & $\mathrm{T}$ \\
\hline
\end{tabular}


Tabela 2 (continuação)

\begin{tabular}{|c|c|c|c|c|c|}
\hline Família/Espécie & $\mathrm{N}^{\circ}$ do Registro & Cris & Sed & $\mathrm{Hab}$ & FV \\
\hline \multicolumn{6}{|l|}{ CUCURBITACEAE } \\
\hline Ceratosanthes trifoliata Cogn. & 45431 & & 1 & $\mathrm{Er} / \mathrm{T}$ & $\mathrm{T}$ \\
\hline \multicolumn{6}{|l|}{ CYPERACEAE } \\
\hline Bulbostylis capillaris (L.) C.B. Clarke & $45651 ; 45430$ & $1 b$ & $19 \mathrm{a}$ & Er & $\mathrm{T}$ \\
\hline Cyperus laxus Lam. & 45434 & & 15 & Er & $\mathrm{T}$ \\
\hline C. uncinulatus Schrad. ex Nees & $45837 ; 45661$ & 2 & $*$ & Er & $\mathrm{T}$ \\
\hline Pycreus propinquus Nees & 45660 & & 5 & Er & $\mathrm{T}$ \\
\hline \multicolumn{6}{|l|}{ DIOSCOREACEAE } \\
\hline Dioscorea ovata Vell. & $45435 ; 45642$ & $8 \mathrm{a}$ & $5 \mathrm{a}$ & $\mathrm{Er} / \mathrm{T}$ & $\mathrm{T}$ \\
\hline Dioscorea sp. & 45436 & 4 & 2 & $\mathrm{Er} / \mathrm{T}$ & $\mathrm{T}$ \\
\hline \multicolumn{6}{|l|}{ EUPHORBIACEAE } \\
\hline Acalypha poiretii Spreng. & $45438 ; 45437$ & $30 \mathrm{a}$ & $7 b$ & Er & $\mathrm{T}$ \\
\hline Bernardia sidoides (Klotzsch) Müll. Arg. & $45440 ; 45439$ & $11 \mathrm{a}$ & $20 \mathrm{a}$ & Er & $\mathrm{T}$ \\
\hline Chamaesyce hyssopifolia (L.) Small & $45441 ; 45840$ & $1 b$ & $10 \mathrm{a}$ & Er & $\mathrm{T}$ \\
\hline Croton glandulosus L. & 45649 & & $*$ & $\mathrm{Er} / \mathrm{S}$ & $\mathrm{T}$ \\
\hline C. lobatus L. & $45443 ; 45442$ & $15 \mathrm{a}$ & $3 b$ & Er & $\mathrm{T}$ \\
\hline Dalechampia scandens L. & 45444 & 9 & & $\mathrm{Er} / \mathrm{T}$ & $\mathrm{T}$ \\
\hline Phyllanthus heteradenius Müll. Arg. & $45445 ; 45446$ & 7 & $*$ & $\mathrm{Er}$ & $\mathrm{T}$ \\
\hline P. niruri L. & $45641 ; 45447$ & $50 \mathrm{a}$ & $66 \mathrm{a}$ & Er & $\mathrm{T}$ \\
\hline Microstachys corniculata (Vahl) Griseb. & 45841 & & 3 & Er & $\mathrm{T}$ \\
\hline \multicolumn{6}{|l|}{ FABACEAE } \\
\hline Aeschynomene viscidula Michx. & 45660 & & $*$ & $\mathrm{Er} / \mathrm{S}$ & $\mathrm{C}$ \\
\hline Crotalaria incana $\mathrm{L}$. & 45639 & & $*$ & $\mathrm{Er} / \mathrm{S}$ & $\mathrm{T}$ \\
\hline Zornia brasiliensis Vogel & $45672 ; 45673$ & $2 b$ & $24 \mathrm{a}$ & $\mathrm{Er}$ & $\mathrm{T}$ \\
\hline Zornia gemella Vogel & 45671 & & 10 & Er & $\mathrm{T}$ \\
\hline \multicolumn{6}{|l|}{ IRIDACEAE } \\
\hline Cipura paludosa Aubl. & 45819 & & $*$ & Er & G \\
\hline \multicolumn{6}{|l|}{ LAMIACEAE } \\
\hline Hypenia salzmannii (Benth.) Harley & 45670 & & 30 & Er & $\mathrm{T}$ \\
\hline Hyptis atrorubens Poit. & $45669 ; 45836$ & $43 b$ & $93 \mathrm{a}$ & $\mathrm{Er}$ & $\mathrm{T}$ \\
\hline \multicolumn{6}{|l|}{ LILIACEAE } \\
\hline Hippeastrum sp. & $45832 ; 45400$ & $7 \mathrm{~b}$ & $36 a$ & Er & G \\
\hline LILIACEAE 1 & 45650 & & 5 & Er & G \\
\hline \multicolumn{6}{|l|}{ MALVACEAE } \\
\hline Herissantia crispa (L.) Brizicky & 45668 & & * & $\mathrm{Er} / \mathrm{S}$ & $\mathrm{C}$ \\
\hline H. tiubae (K. Schum.) Brizicky & $45666 ; 45667$ & $50 \mathrm{a}$ & $23 b$ & $\mathrm{Er} / \mathrm{S}$ & $\mathrm{C}$ \\
\hline Pseudomalachra sp. & $45665 ; 45664$ & $22 b$ & $46 a$ & $\mathrm{Er} / \mathrm{S}$ & $\mathrm{T}$ \\
\hline Sida cordifolia L. & $45638 ; 45662$ & $10 \mathrm{a}$ & $9 a$ & $\mathrm{Er} / \mathrm{S}$ & $\mathrm{T}$ \\
\hline \multicolumn{6}{|l|}{ MOLLUGINACEAE } \\
\hline Mollugo verticillata $\mathrm{L}$. & $45637 ; 45636$ & $56 a$ & $75 a$ & $\mathrm{Er}$ & $\mathrm{T}$ \\
\hline \multicolumn{6}{|l|}{ MORACEAE } \\
\hline Dorstenia sp. & 45635 & 6 & & Er & G \\
\hline \multicolumn{6}{|l|}{ NYCTAGINACEAE } \\
\hline Boerhavia coccinea Mill. & $45465 ; 45464$ & $11 \mathrm{a}$ & $15 \mathrm{a}$ & Er & $\mathrm{T}$ \\
\hline \multicolumn{6}{|l|}{ PAPAVERACEAE } \\
\hline Argemone mexicana $\mathrm{L}$. & 45826 & & * & Er & $\mathrm{T}$ \\
\hline \multicolumn{6}{|l|}{ PASSIFLORACEAE } \\
\hline Passiflora foetida $\mathrm{L}$. & 45463 & & $*$ & $\mathrm{Er} / \mathrm{T}$ & $\mathrm{T}$ \\
\hline \multicolumn{6}{|l|}{ PHYTOLACACEAE } \\
\hline Microtea paniculata Moq. & $45461 ; 45462$ & $75 a$ & $4 b$ & $\mathrm{Er}$ & $\mathrm{T}$ \\
\hline POACEAE & & & & & \\
\hline Axonopus capillaris (Lam.) Chase & $45844 ; 45648$ & $4 \mathrm{~b}$ & $39 a$ & Er & $\mathrm{T}$ \\
\hline Dactyloctenium aegyptium (L.) Willd. & $45460 ; 45663$ & $3 b$ & $19 \mathrm{a}$ & Er & $\mathrm{T}$ \\
\hline Digitaria sanguinalis (L.) Scop. & $45459 ; 45652$ & 7 & $*$ & Er & $\mathrm{T}$ \\
\hline Enteropogon mollis (Nees) Clayton & $45646 ; 45659$ & 4 & $*$ & Er & $\mathrm{T}$ \\
\hline Eragrostis acutiflora (Kunth) Nees & 45656 & & 11 & Er & $\mathrm{T}$ \\
\hline E. ciliaris $(\mathrm{L}.) \mathrm{R}$. Br. & $45457 ; 45458$ & $19 b$ & $45 \mathrm{a}$ & Er & $\mathrm{T}$ \\
\hline E. pilosa (L.) P. Beauv. & 45828 & & 9 & Er & $\mathrm{T}$ \\
\hline E. tenella (L.) P. Beauv. ex Roem. \& Schult. & 45456 & $*$ & & Er & $\mathrm{T}$ \\
\hline E. unioloides (Retz.) Nees ex Steud. & $45645 ; 45654$ & $*$ & $*$ & Er & $\mathrm{T}$ \\
\hline Panicum trichoides Sw. & $45644 ; 45657$ & $64 a$ & $49 \mathrm{a}$ & Er & $\mathrm{T}$ \\
\hline Tragus berteronianus Schult. & $45454 ; 45455$ & $1 b$ & $8 \mathrm{a}$ & Er & $\mathrm{T}$ \\
\hline
\end{tabular}


106 Silva, Araújo \& Ferraz: Estudo florístico do componente herbáceo e relação com solos em áreas de caatinga...

\begin{tabular}{|c|c|c|c|c|c|}
\hline Família/Espécie & $\mathrm{N}^{\circ}$ do Registro & Cris & Sed & Hab & FV \\
\hline \multicolumn{6}{|l|}{ PORTULACACEAE } \\
\hline Portulaca elatior Mart. ex Rohrb. & $45839 ; 45830$ & $41 b$ & $68 \mathrm{a}$ & $\mathrm{Er}$ & $\mathrm{T}$ \\
\hline P. mucronata Link & $45835 ; 45831$ & $28 b$ & $63 a$ & $\mathrm{Er}$ & $\mathrm{T}$ \\
\hline P. oleracea $\mathrm{L}$. & $45834 ; 45829$ & $6 a$ & $3 a$ & Er & $\mathrm{T}$ \\
\hline P. umbraticola Kunth & 45833 & $*$ & & Er & $\mathrm{T}$ \\
\hline \multicolumn{6}{|l|}{ RHAMNACEAE } \\
\hline Crumenaria decumbens Mart. & 45453 & 3 & & $\mathrm{Er}$ & $\mathrm{T}$ \\
\hline \multicolumn{6}{|l|}{ RUBIACEAE } \\
\hline Mitracarpus scabrellus Benth. & 47684 & 10 & & Er & $\mathrm{T}$ \\
\hline \multicolumn{6}{|l|}{ RUBIACEAE } \\
\hline Richardia grandiflora (Cham. \& Schltdl.) Steud. & $45848 ; 45847$ & $31 b$ & $87 \mathrm{a}$ & $\mathrm{Er}$ & $\mathrm{T}$ \\
\hline Staelia virgata (Link ex Roem. \& Schult.) K. Schum. & $45846 ; 45845$ & $23 b$ & $89 \mathrm{a}$ & Er & $\mathrm{T}$ \\
\hline \multicolumn{6}{|l|}{ SAPINDACEAE } \\
\hline Cardiospermum sp. & $45451 ; 45452$ & $19 \mathrm{a}$ & $16 a$ & $\mathrm{Er} / \mathrm{T}$ & $\mathrm{C}$ \\
\hline \multicolumn{6}{|l|}{ SCROPHULARIACEAE } \\
\hline Angelonia cornigera Hook. & $45827 ; 45822$ & $*$ & $*$ & Er & $\mathrm{T}$ \\
\hline Tetraulacium veroniciforme Turcz. & 45450 & $2 b$ & $11 \mathrm{a}$ & $\mathrm{Er}$ & $\mathrm{T}$ \\
\hline \multicolumn{6}{|l|}{ SOLANACEAE } \\
\hline Physalis neesiana Sendtn. & 45449 & 1 & & $\mathrm{Er}$ & $\mathrm{T}$ \\
\hline Schwenckia americana var. angustifolia J.A. Schmidt & 45823 & 8 & & Er & $\mathrm{T}$ \\
\hline \multicolumn{6}{|l|}{ STERCULIACEAE } \\
\hline Ayenia erecta Mart. ex K. Schum. & 45448 & & 10 & Er & $\mathrm{T}$ \\
\hline Waltheria macropoda Turcz. & 45838 & & $*$ & Er & $\mathrm{C}$ \\
\hline W. rotundifolia Schrank & 45647 & & 3 & $\mathrm{Er}$ & $\mathrm{T}$ \\
\hline \multicolumn{6}{|l|}{ TILIACEAE } \\
\hline Corchorus hirtus L. & $45677 ; 45678$ & $4 \mathrm{a}$ & $12 \mathrm{a}$ & Er & $\mathrm{T}$ \\
\hline \multicolumn{6}{|l|}{ TURNERACEAE } \\
\hline Turnera ulmifolia $\mathrm{L}$. & 45676 & & 1 & $\mathrm{Er} / \mathrm{S}$ & $\mathrm{T}$ \\
\hline \multicolumn{6}{|l|}{ URTICACEAE } \\
\hline Pilea hyalina Fenzl & 45675 & 8 & & Er & $\mathrm{T}$ \\
\hline \multicolumn{6}{|l|}{ VERBENACEAE } \\
\hline Stachytarpheta sanguinea Mart. & 45674 & & $*$ & $\mathrm{Er}$ & $\mathrm{T}$ \\
\hline Total de espécies & & 69 & 78 & & \\
\hline
\end{tabular}

próxima da faixa de variação (14 a 34 famílias e 21 a 73 espécies) que vem sendo registrada em outros estudos sobre plantas herbáceas da caatinga (Rodal et al. 2005; Reis et al. 2006; Feitoza et al. 2008; M.O.M. Feitoza, dados não publicados).

As famílias da área sedimentar com maior número de espécies foram: Poaceae (10), Euphorbiaceae (8), Asteraceae (5), Capparaceae (4), Convolvulaceae (4), Cyperaceae (4), Fabaceae (4) e Malvaceae (4) e juntas somaram 55\% do total de espécies. Por outro lado, nesta mesma área, 53\% das famílias apresentaram apenas uma espécie. Na área cristalina, as famílias com mais espécies foram Poaceae (9), Euphorbiaceae (7), Convolvulaceae (5), Portulacaceae (4), Bromeliaceae (3), Malvaceae (3) e Rubiaceae (3). Juntas corresponderam a $49 \%$ do total de espécies, enquanto $48 \%$ das famílias apresentaram apenas uma espécie (Tab. 2). Com exceção de Capparaceae, as demais famílias são geralmente apontadas como de elevada riqueza de espécies no componente herbáceo da caatinga (Araújo et al. 2002a;
Araújo 2003; Araújo et al. 2005; Rodal et al. 2005; Reis et al. 2006; Feitoza et al. 2008; M.O.M. Feitoza, dados não publicados).

Os gêneros Eragrostis (4 e 3 espécies) e Portulaca (3 e 4 espécies) foram os mais ricos nas áreas sedimentar e cristalina (Tab. 2). Além desses, merecem destaque Cleome, na área sedimentar e Ipomoea na área cristalina, com três espécies cada (Tab. 2). Estes gêneros geralmente são apontados como os mais ricos em outros estudos sobre o componente herbáceo da caatinga (Araújo et al. 2002a; Reis et al. 2006).

Entre as áreas sedimentar e cristalina, o percentual de famílias em comum foi elevado (64\%). Este fato, aliado à pequena diferença na diversidade das famílias mais ricas, sugere que esse nível taxonômico não permite visualizar diferenças na composição florística de áreas próximas, mesmo que apresentem solos distintos (Tab. 1). De acordo com Lemos \& Rodal (2002), o nível taxonômico de família também não foi um bom caráter para individualizar conjuntos florísticos no componente 
lenhoso da caatinga, ao compararem famílias de elevada riqueza de espécies arbóreas de muitas áreas já estudadas.

Em relação à flora total das áreas, o número comum de espécies foi de 52 (55\%), sendo sete observadas fora das parcelas (Tab. 2). As 52 espécies em comum correspondem a $67 \%$ das espécies da área sedimentar (solos arenosos) e a 75\% das espécies da área cristalina (solos pedregosos). A similaridade florística entre as áreas pelo índice se Sfrensen foi de 75\%. Assim, apesar das diferenças nas características do solo, a semelhança florística entre áreas foi elevada. Possivelmente, essa semelhança seja explicada pela proximidade entre as áreas. Em outras palavras, proximidades entre áreas seria um fator capaz de atenuar a influência que o tipo de solo teria sobre a composição de herbáceas instaladas na área.

Por outro lado, excetuando as espécies observadas e as que ocorreram em menos de cinco parcelas, apenas 15 espécies de um total de 40 não apresentaram diferenças de freqüência nas parcelas entre as áreas. O restante deste total (25 espécies) apresentou diferença quanto à ocorrência, sendo 18 (72\%) presentes em um maior número de parcelas na área sedimentar e sete (28\%) nas parcelas da área cristalina (Tab. 2). Assim, apesar da influência dos solos sobre a composição de ervas poder ser atenuada pela proximidade entre as áreas, não parece que o arranjo no espaço de algumas delas o seja. Isto também foi evidenciado na análise de agrupamento (Fig. 1) que indicou a ocorrência de grupos florísticos distintos, pois apenas três parcelas da área cristalina agruparam-se com as da área sedimentar, mostrando que apesar da elevada similaridade os arranjos florísticos entre as parcelas amostradas foram mais semelhantes dentro de cada área que entre áreas. As espécies Hyptis atrorubens e Staelia virgata foram responsáveis pela ligação das três parcelas do cristalino ao grupo das parcelas da área sedimentar, pois apesar de comum às duas áreas, apresentavam freqüência significativa na área sedimentar (Tab. 2). Logo, se admitirmos que as condições de temperatura, umidade relativa e precipitação anual tenham sido semelhantes entre as áreas, devido à proximidade das mesmas, então é possível admitir que, ao menos em parte, os solos podem ser considerados como tendo influência na distribuição de algumas espécies herbáceas da caatinga, justificando as diferenças nas freqüências registradas (Tab. 2).

Das espécies amostradas apenas no trecho sedimentar Centratherum punctatum, Herissantia crispa e Turnera ulmifolia já haviam sido registradas em outras áreas de caatinga do cristalino por Reis et al. (2006) e Feitoza et al. (2008), mas Ayenia erecta, Acanthospermum hispidum, Aeschynomene viscidula,
Crotalaria incana, Ceratosanthes trifoliata, Chamaecrista trichopoda, Cleome guianensis, Croton glandulosus, Cyperus laxus, Dactylaena micrantha, Eragrostis acutiflora, E. pilosa, Hypenia salzmannii, Microstachys corniculata, Pycreus propinquus, Stachytarpheta sanguinea, Waltheria macropoda, Waltheria rotundifolia e Zornia gemella não apresentam registro de ocorrência quando comparadas a outras áreas cristalinas da vegetação da caatinga (Araújo et al. 2002a; Reis et al. 2006; Feitoza, et al. 2008). De igual forma, 13 espécies amostradas apenas na área cristalina não haviam sido registradas em áreas sedimentares: Crumenaria decumbens, Dalechampia scandens, Encholirium spectabile, Eragrostis tenella, Hohenbergia catingae, Ipomoea aristolochiifolia, Matelea maritima subsp.

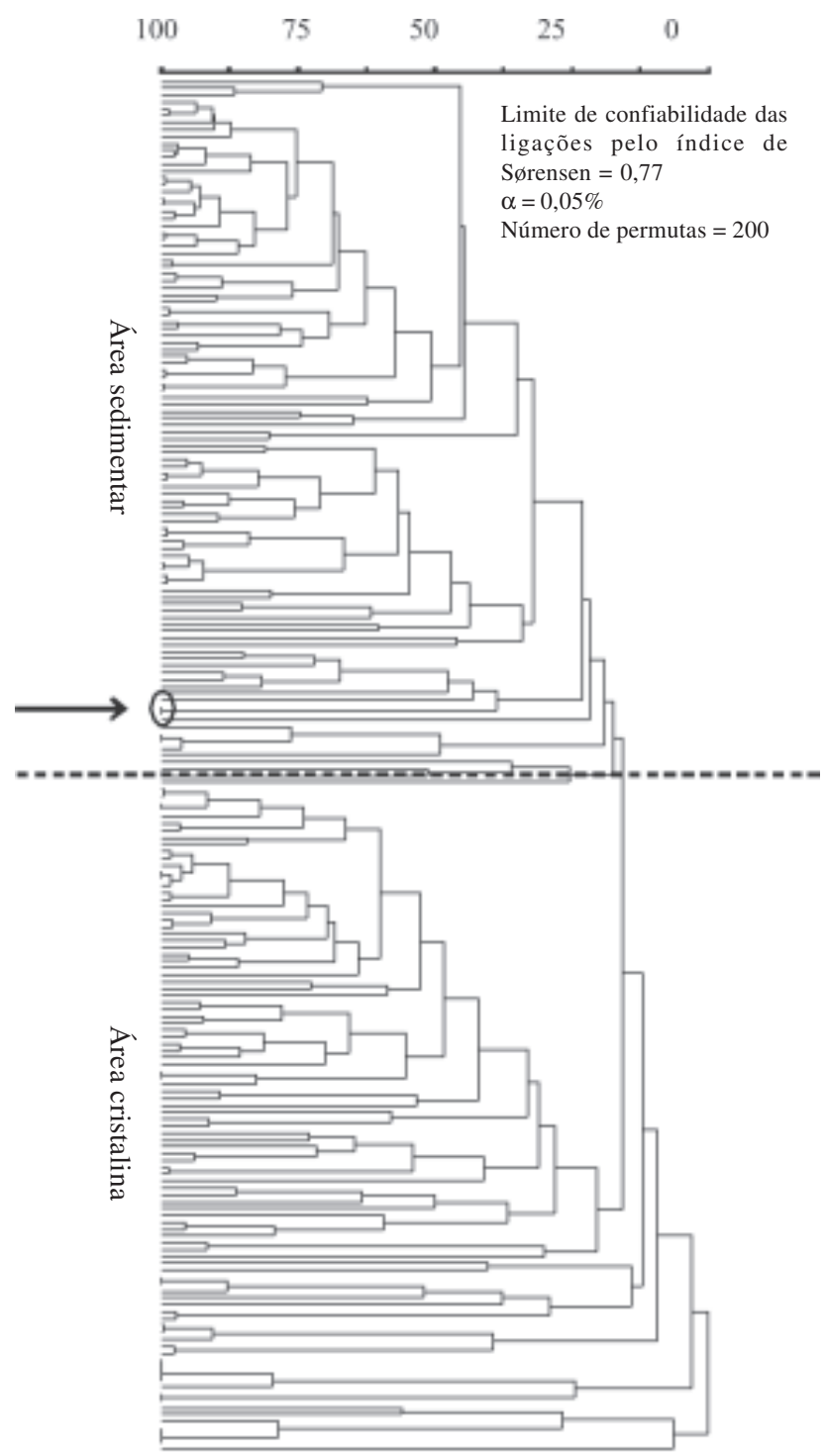

Figura 1. Similaridade florística entre as áreas sedimentar e cristalina de uma área de caatinga, Petrolândia, PE, Brasil. A seta indica a posição das três parcelas da área cristalina que ocorreram agrupadas com as parcelas da área sedimentar. 
ganglinosa, Melocactus bahiensis, Neoglaziovia variegata, Physalis neesiana, Pilea hyalina, Portulaca umbraticola e Schwenckia americana var. angustifolia (Rodal et al. 1999; Araújo et al. 2002a; Reis et al. 2006; M.O.M. Feitoza, dados não publicados). Diante do exposto, apesar da elevada similaridade florística entre as áreas foi possível identificar grupos de espécies exclusivos de cada uma delas.

Os percentuais de formas de vidas identificados entre as áreas sedimentar e cristalina foram similares, com predominância da forma terófita. A área sedimentar esteve representada por 65 terófitas, nove caméfitas, sendo uma suculenta e quatro geófitas. Já a área cristalina esteve representada por 53 terófitas, 11 caméfitas, sendo duas suculentas e quatro geófitas (Fig. 2). O percentual de terófitas registrado é elevado em relação ao encontrado em outras formações vegetacionais (Conceição \& Giulietti 2002; Garcia \& Pirani 2005), mas confirma que a forma de vida terófita é a principal estratégia de escape à seca adotada pelas plantas herbáceas da caatinga (Araújo et al. 2002a; Pessoa et al. 2004; Costa et al. 2007; Feitoza et al. 2008).

A forma de vida hemicriptófita não foi observada em nenhuma das áreas deste estudo, diferindo do encontrado por Costa et al. (2007) e Pessoa et al. (2004) ao registrar que $17 \%$ e $26 \%$ das espécies de áreas de caatinga do Ceará e de Pernambuco, respectivamente, apresentavam a forma de vida hemicriptófita como estratégia de escape à estação desfavorável. Já o percentual de geófitas (cerca de 5\%) foi próximo ao registrado por Pessoa et al. 2004 (4\%), mas difere do observado por Costa et al. (2007) que não registraram a ocorrência desta forma de vida em seu estudo. Fanerófitas e epífitas não foram observadas nas comunidades herbáceas estudadas, apesar de existir registro de ocorrência de espécies destas formas de vida

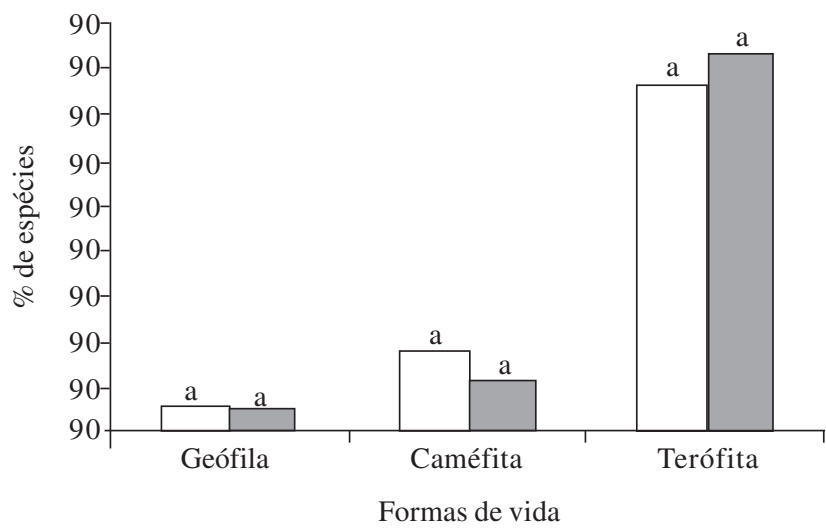

Figura 2. Percentual de espécies por formas de vida nas áreas sedimentar e cristalina em uma área de caatinga, Petrolândia, PE, Brasil (letras semelhantes em uma mesma categoria de forma de vida, indicam nenhuma diferença entre áreas pelo teste do Quiquadrado). ( $\square=$ Área cristalina; $\square=$ Área sedimentar). na vegetação da caatinga (Araújo et al. 2002a; Araújo et al. 2002b; França et al. 2006). Estes dados mostram que apesar da forma terófita ser predominante, as áreas de caatinga podem diferir quanto aos percentuais das demais formas de vida que ocorram nas mesmas. Vale comentar que as caméfitas Encholirium spectabile, Hohenbergia catingae, Melocactus bahiensis e Neoglaziovia variegata ocorriam predominantemente sobre as rochas e marcaram a fisionomia da vegetação da área cristalina.

Com base em consultas aos herbários Professor Vasconcelos Sobrinho (PEUFR) e Dárdano de Andrade Lima (IPA) em Pernambuco foi constatado que apenas Angelonia cornigera (Scrophulariaceae), Ayenia erecta (Sterculiaceae), Bulbostylis capillaris (Cyperaceae), Evolvulus frankenioides (Convolvulaceae) e Zornia brasiliensis (Fabaceae) haviam sido coletadas no município de Petrolândia, mostrando que a flora herbácea daquele município era pouco conhecida. Portanto, o esforço de coleta deste estudo reduziu a insuficiência de conhecimento sobre a flora herbácea do município.

Alguns dos estudos florísticos (Souza \& Giulietti 1990; Sales et al. 1998; Rodal et al. 1999; Costa et al. 2007; Albuquerque et al. 2007) e fitossociológicos (M.F.A.V. Santos, dados não publicados; Alcoforado Filho et al. 2003) do componente lenhoso da caatinga, também listaram as ervas presentes nas áreas. Estas listas, somadas às listas florísticas disponibilizadas nos estudos realizados especificamente sobre o componente herbáceo (Pereira et al.1989; Araújo et al. 2002a; Pessoa et al. 2004; Reis et al. 2006; Araújo et al. 2005; Feitoza et al. 2008; M.O.M. Feitoza, dados não publicados), mostram que a flora herbácea da caatinga reunia, até o momento, um total de 568 espécies. Das espécies identificadas neste estudo, 19 ainda não haviam sido listadas. Todavia, vale salientar, que o número de 687 espécies de herbáceas deve estar ainda subestimado, pois tal indicativo de riqueza da flora herbácea advém dos poucos estudos realizados, basicamente em Pernambuco e algumas das listas têm espécies identificadas apenas ao nível de gênero e família, não tendo sido incluídas nesta primeira soma.

Por fim, este estudo conclui que o tipo de solo parece não ser um fator decisivo na similaridade florística, hábito e proporção de formas de vida das herbáceas da caatinga quando as áreas são próximas, mas pode influenciar a ocorrência e a freqüência de algumas espécies.

\section{Agradecimentos}

À senhora Maria Elita Ramalho Leal e toda a sua família, que residem no distrito Mundo Novo, pela hospitalidade; ao Programa de Pós-Graduação em Botânica da Universidade Federal Rural de Pernambuco; 
ao CNPq, pela concessão de bolsas de mestrado e de produtividade em pesquisa; a Josiene Maria Falcão Fraga dos Santos, pelo apoio no tratamento dos dados e aos botânicos: Afrânio Fernandes, Alexa Araújo, Ana Paula Fortuna Peres, André Laurênio de Melo, Iranildo Melo, Ângela Maria Miranda, Elnatan Bezerra, Jéferson Rodrigues, Josafá Siqueira, José Alves Siqueira, Marcos J. Silva, Maria Bernadete Costa e Silva, Maria de Fátima A. Lucena, Rita Pereira, Roxana Pereira e Suzene Izídio da Silva que auxiliaram na identificação botânica.

\section{Referências bibliográficas}

Albuquerque, U.P.; Medeiros, P.M.; Almeida, A.L.S.; Monteiro, J.M.; Lins Neto, E.M.F.; Melo, J.G. \& Santos, J.P. 2007. Medicinal plants of the caatinga (semi-arid) vegetation of NE Brazil: a quantitative approach. Journal of Ethnopharmacology (doi:10.1016/j.jep.2007.08.017).

Alcoforado Filho, F.G.; Sampaio, E.V.S.B. \& Rodal, M.J.N. 2003. Florística e fitossociologia de um remanescente de vegetação caducifólia espinhosa arbórea em Caruaru, Pernambuco. Acta Botanica Brasilica 17: 287-303.

Andrade-Lima, D. 1981. The caatingas dominium. Revista Brasileira de Botânica 4: 149-163.

Araújo, E.L.; Sampaio, E.V.S.B. \& Rodal, M.J.N. 1995. Composição florística e estrutura em três áreas de caatinga de Pernambuco. Revista Brasileira de Biologia 55: 595-607.

Araújo, E.L.; Silva, S.I. \& Ferraz, E.M.N. 2002a. Herbáceas da caatinga de Pernambuco. Pp. 183-206. In: J.M. Silva \& M. Tabarelli (orgs.). Diagnóstico da biodiversidade do estado de Pernambuco. Recife, SECTMA.

Araújo, E.L.; Freitas, A.M.M.; Silveira, A.P. \& Pires, M.F.O. 2002b. Hospedeiras lenhosas de espécies de Tillandsia na caatinga em Pernambuco. Pp. 237-244. In: J.M. Silva \& M. Tabarelli (orgs.). Diagnóstico da Biodiversidade do estado de Pernambuco. Recife, SECTMA.

Araújo, E.L. 2003. Diversidade de herbáceas na vegetação da caatinga. Pp. 82-84. In: E.A.G. Jardim; M.N.C. Bastos \& J.U.M. Santos (eds.). Desafios da Botânica brasileira no novo milênio: inventário, sistematização e conservação da diversidade vegetal. Belém, Sociedade Botânica do Brasil.

Araújo, E.L.; Silva, K.A.; Ferraz, E.M.N.; Sampaio, E.V.S.B. \& Silva, S.I. 2005. Diversidade de herbáceas em microhabitats rochoso, plano e ciliar em uma área de caatinga, Caruaru, PE, Brasil. Acta Botanica Brasilica 19: 285-294.

Araújo, E.L.; Castro, C.C. \& Albuquerque, U.P. 2007. Dynamics of Brazilian Caatinga - A Review Concerning the Plants, Environment and People. Functional Ecosystems and Communities 1: 15-28.

Araújo Filho, J.A.; Carvalho, F.C.; Garcia, R. \& Sousa, R.A. 2002. Efeitos da manipulação da vegetação lenhosa sobre a produção e compartimentalização da fitomassa pastável de uma caatinga sucessional. Revista Brasileira de Zootecnia 31: 11-19.

Brasil, Ministério das Minas e Energia. Projeto RADAMBRASIL. 1983. Folhas SC 24/25 - Aracaju/Recife. Geologia, geomorfologia, pedologia, vegetação e uso potencial da terra. Rio de Janeiro, Ministério da Minas e Energia (Levantamento de Recursos Naturais, 30).
Brummitt, R.K. \& Powell, C.E. 1992. Authors of plant names. Kew, Royal Botanical Garden.

Conceição, A.A. \& Giulietti, A.M. 2002. Composição florística e aspectos estruturais de campo rupestre em dois platôs do Morro do Pai Inácio, Chapada Diamantina, Bahia, Brasil. Hoehnea 29: 37-48.

Costa, R.C.; Araújo, F.S. \& Lima-Verde, L.W. 2007. Flora and life-form spectrum in an area of deciduous thorn woodland (caatinga) in northeastern, Brazil. Journal of Arid Environments 68: 237-247.

Cronquist, A. 1988. An integrated system of classification of flowering plants. New York, Columbia University Press.

Feitoza, M.O.M.; Araújo, E.L.; Sampaio, E.V.S.B. \& Kiill, L.H.P. 2008. Fitossociologia e danos foliares ocorrentes na comunidade herbácea de uma área de caatinga em Petrolina, PE. Pp. 7-30. In: A.N. Moura; E.L. Araújo \& U.P. Albuquerque (eds.). Biodiversidade, potencial econômico e processos eco-fisiológicos em ecossistemas nordestinos. Recife, Comunigraf/Nupea.

Ferraz, E.M.N.; Rodal, M.J.N.; Sampaio, E.V.S.B. \& Pereira, R.C.A. 1998. Composição florística em trechos de vegetação de caatinga e brejo de altitude na região do Vale do Pajeú, Pernambuco. Revista Brasileira de Botânica 21: 7-15.

FIAM/DI (Recife). 1986. Enciclopédia dos municípios do interior de Pernambuco. Recife, FIAM/DI.

França, F.; Melo, E. \& Gonçalves, J.M. 2006. Aspectos da diversidade da vegetação no topo de um inselbergue no semiárido da Bahia, Brasil. Sitientibus Série Ciências Biológicas 6: $30-35$.

Fuller, D.O. 1999. Canopy phenology of some mopane and miombo woodlands in eastern Zambia. Global Ecology and Biogeography 8: 199-209.

Garcia, R.J.F. \& Pirani, J.R. 2005. Análise florística, ecológica e fitogeográfica do Núcleo Curucutu, Parque Estadual da Serra do Mar (São Paulo, SP), com ênfase nos campos junto à crista da Serra do Mar. Hoehnea 32: 1-48.

Jacomine, P.K.T.; Cavalcanti, A.C.; Burgos, N.; Pessoa, S.C.P. \& Silveira, C.O. 1973. Levantamento exploratório - reconhecimento de solos do Estado de Pernambuco: descrição de perfis de solos e análises. v.1. Recife, SUDENE (Boletim Técnico, 26).

Knoop, W.T. \& Walker, B.H. 1985. Interactions of woody and herbaceous vegetation in a southern African savana. Journal of Ecology 73: 235-253.

Krebs, C.J. 1989. Ecological methodology. New York, Harper \& Row Publishers.

Lemos, J.R. \& Rodal, M.J.N. 2002. Fitossociologia do componente lenhoso em um trecho de vegetação de caatinga no Parque Nacional Serra da Capivara, Piauí, Brasil. Acta Botanica Brasilica 16: 23-42.

Lorenzon, M.C.A.; Matrangolo, C.A. \& Schoereder, J.H. 2003. Flora visitada pelas abelhas eussociais (Hymenoptera, Apidae) na Serra da Capivara, em Caatinga do Sul do Piauí. Neotropical Entomology 32: 27-36.

McCune, B. \& Mefford, M.J. 1999. PC-ORD Versão 4.0. Multivariate analysis of ecological data; Users guide. Gleneden Beach, MJM Software Design.

Mori, S.A.; Silva, L.A.M. \& Lisboa, G. 1989. Manual de manejo do herbário fanerogâmico. Ilhéus, Centro de Pesquisa do Cacau. 
110 Silva, Araújo \& Ferraz: Estudo florístico do componente herbáceo e relação com solos em áreas de caatinga...

Oliveira, M.E.A.; Sampaio, E.V.S.B.; Castro, A.A.J.F. \& Rodal, M.J.N. 1997. Flora e fitossociologia de uma área de transição carrasco-caatinga de areia em Padre Marcos. Piauí. Naturalia 22: $131-150$.

Perazzo, G.M. 2002. Perfil do saneamento ambiental em 29 municípios da área de Xingó. Recife, Imprensa Universitária.

Pereira, R.M.A.; Araújo Filho, J.A.; Lima, R.V.; Paulino, F.D.G.; Lima, A.O.N. \& Araújo, Z. 1989. Estudos fenológicos de algumas espécies lenhosas e herbáceas da caatinga. Ciências Agronômicas 20: 11-20.

Pessoa, L.M.; Rodal, M.J.N.; Lins e Silva, A.C.B.L. \& Costa, K.C.C. 2004. Levantamento da flora herbácea em um trecho de caatinga, RPPN Maurício Dantas, Betânia/Floresta, Pernambuco. Revista Nordestina de Biologia 18: 27-53.

Peters, D.P.C. 2002. Plant species dominanc at a grasslandShrubland ecotone: and individual-based gap dynamics model of herbaceous and species woody. Ecological Modeling 152: 5-32.

Prado, D. 2003. As caatingas da América do Sul. Pp. 3-73. In: R.I. Leal; M. Tabarelli \& J.M.C. Silva (eds.). Ecologia e Conservação da caatinga. Recife, Editora Universitária.

Raunkiaer, C. 1934. Life forms of plants and statistical plant geography. Oxford, Clarendon Press.

Reis, A.M.S.; Araújo, E.L.; Ferraz, E.M.N. \& Moura, A.N. 2006. Inter-annual variations in the floristic and population structure of an herbaceous community of "caatinga" vegetation in Pernambuco, Brazil. Acta Botanica Brasilica 29: 497-508.

Rodal, M.J.N.; Nascimento, L.M. \& Melo, A.L. 1999. Composição florística de um trecho de vegetação arbustiva caducifólia, no município de Ibimirim, Pernambuco, Brasil. Acta Botanica Brasilica 13: 15-28.
Rodal, M.J.N.; Lins e Silva, A.C.B.; Cavalcanti, A.D.C. \& Pessoa, L.M. 2005. Vegetação e flora fanerogâmica da área de Betânia, Pernambuco. Pp. 141-168. In: F.S. Araújo; M.J.N. Rodal \& M.R.V. Barbosa (eds.). Análise das variações da biodiversidade do bioma caatinga: suporte e estratégias regionais de conservação. Fortaleza, Ministério do Meio Ambiente.

Sales, M.F.; Mayo, S.J. \& Rodal, M.J.N. 1998. Plantas vasculares das florestas serranas de Pernambuco - Um checklist da flora ameaçada dos brejos de altitude. Recife, Imprensa Universitária.

Sampaio, E.V.S.B. \& Gamarra-Rojas, C.F.L. 2003. A vegetação lenhosa das ecorregiões da caatinga. Pp. 85-90. In: E.A.G. Jardim; M.N.C. Bastos \& J.U.M. Santos (eds.). Desafios da Botânica brasileira no novo milênio: inventário, sistematização e conservação da diversidade vegetal. Belém, Sociedade Botânica do Brasil.

Sampaio, E.V.S.B. 2003. A caracterização da caatinga e fatores ambientais que afetam a ecologia das plantas lenhosas. Pp. 129-142. In: V. Claudino-Sales (ed.). Ecossistemas brasileiros: manejo e conservação. Fortaleza, Expressão Gráfica.

Souza, V.C. \& Giulietti, A.M. 1990. Scrophulariaceae de Pernambuco. Boletim de Botânica 12: 185-209.

Souza, M.J.N.; Martins, M.L.R.; Soares, Z.M.L.; Freitas Filho, M.R.; Almeida, M.A.G.; Pinheiro, F.S.A.; Sampaio, M.A.B.; Carvalho, G.M.B.S.; Soares, A.M.L.; Gomes, E.C.B. \& Silva, R.A. 1994. Redimensionamento da região semi-árida do Nordeste do Brasil. Fortaleza, Fundação Esquel do Brasil.

Zar, J.H. 1996. Bioestatistical Analysis. New Jersey, Prentice Hall. 